\title{
Multi-Scale Salient Features for Analyzing 3D Shapes
}

\author{
Yong-Liang Yang ${ }^{1,2}$ (杨永亮) and Chao-Hui Shen ${ }^{2}$ (沈超慧) \\ ${ }^{1}$ King Abdullah University of Science and Technology, Thuwal, Saudi Arabia \\ ${ }^{2}$ Tsinghua National Lab for Informatics Science \& Technology, Tsinghua University, Beijing 100084, China \\ E-mail: yongliang.yang@kaust.edu.sa; chench08@mails.tsinghua.edu.cn
}

Received September 5, 2012; revised September 13, 2012.

\begin{abstract}
Extracting feature regions on mesh models is crucial for shape analysis and understanding. It can be widely used for various 3D content-based applications in graphics and geometry field. In this paper, we present a new algorithm of extracting multi-scale salient features on meshes. This is based on robust estimation of curvature on multiple scales. The coincidence between salient feature and the scale of interest can be established straightforwardly, where detailed feature appears on small scale and feature with more global shape information shows up on large scale. We demonstrate this kind of multi-scale description of features accords with human perception and can be further used for several applications as feature classification and viewpoint selection. Experiments exhibit that our method as a multi-scale analysis tool is very helpful for studying $3 \mathrm{D}$ shapes.
\end{abstract}

Keywords multi-scale, salient feature, shape analysis

\section{Introduction}

Due to the fast development of $3 \mathrm{D}$ scanning and modeling technology, triangular meshes are now widely used in computer graphics. Objects with fruitful surface details can be well captured and constructed into mesh form. The interest in analyzing the geometric information of meshes is ever increasing. This is the most important step for a variety of applications in computer graphics, computer vision and geometric modeling, such as shape retrieval, shape alignment, feature preserved simplification.

In shape analysis, the key is how to define intrinsic features which can well represent the model's characteristic. To ensure the intrinsic property, the features are often required to be invariant under rigid transformation and uniform scaling. Moreover, the extracted feature should be discriminative to other models especially with different types. Based on different feature definitions, shape analysis methods can be generally classified into two categories: global and local ${ }^{[1]}$. The former one focuses on describing the entire shape of the model with a so-called "shape descriptor". The methodology of 3D statistics like shape distribution and histogram is usually involved, while local geometric details are not concerned much. On the other hand, local methods define features based on local surface properties. Curvature and its related quantities are often used here.

There have been several publications about determining saliency or extracting salient features on meshes in the recent years. [2] defines a measure of mesh saliency using a center-surround operator on Gaussianweighted mean curvatures. This work incorporates insights from human perception, while the extraction of interesting feature parts is not their concern. [3] defines salient feature as region with high importance and nontrivial local shapes. It proposed to extract salient features based on curvature from local fitting, but there is no scale specialty of features considered here. Shilane et $a ._{.}^{[4]}$ presented a novel method to select regions that distinguish a shape by not only judging the shape itself. It is based on performing a shape-based search using each region as a query into a database. This method can reasonably select the regions which successfully discriminate the model with others, but the precondition is the availability of a shape retrieval environment. Recently, Chen et al. ${ }^{[5]}$ investigated the socalled "schelling points" on 3D surface. These points have to be manually selected by the users beforehand

Regular Paper

This work was supported by the National Basic Research 973 Program of China under Grant No. 2011CB302203, the National Natural Science Foundation of China under Grant No. 61120106007, and the National High Technology Research and Development 863 Program of China under Grant No. 2012AA011801.

* The preliminary version of the paper was published in the Proceedings of the 2012 Computational Visual Media Conference.

** The test models used in this paper are courtesy of the Stanford 3D scanning repository and the AIM@SHAPE shape repository.

C 2012 Springer Science + Business Media, LLC \& Science Press, China 
on a training dataset. Then features can be predicted on new shapes based on the prior knowledge.

In this paper, we present a method of extracting salient geometric features on multiple scales. It is more likely to analyze local shape properties, while global shape information is taken into account when the scale of interest becomes large (see Fig.1). Although the definition of salient feature is also based on curvature and its variance, the curvature estimation is performed in a multi-scale way. The salient features extracted on different scales represent different levels of surface details. We show that the scale specialty of salient features can help us to understand the surface shape more comprehensively.

The main contributions of our paper can be summarized as the following:

- We use multi-scale curvature estimation as an analysis tool in salient feature extraction. An extended curvature-driven local surface descriptor building method is introduced.

- Two novel applications based on multi-scale salient features are presented for comprehensively studying different levels of surface details.

This paper is an extended version of [6]. More discussions and experimental results are presented. The rest of the paper is organized as follows. In Section 2, recent work related to multi-scale salient feature extraction is discussed. In Section 3, we will describe the procedure of multi-scale salient feature extraction in detail. Two interesting applications which benefit from our method will be presented in Section 4. Finally, we conclude our paper and discuss some of the future work in Section 5.

\section{Related Work}

\subsection{Curvature Estimation on Meshes}

Differential invariants such as curvatures and its higher order derivatives play a very important role in local shape investigations. As the extensive availability of triangular meshes in computer graphics, there have been significant progresses on estimating curvatures on discrete mesh representation ${ }^{[7]}$. Curvature estimation methods generally fall into two classes: discrete differential geometry based and using proxies from local fitting. In [8] and [9], a novel method of estimating curvatures from an integral invariant viewpoint was presented. The basic idea is to use principal component analysis (PCA) of the local surface point's spherical neighborhood to enhance the robustness and consistency. The advantage of this method is a naturally defined multi-scale property, i.e., the scale of curvature is just the radius of the estimating local neighborhood. It provides a possible way for multi-scale shape analysis.

\subsection{Mesh Saliency and Salient Feature}

Saliency has been studied on 2D images for years and has many applications such as image retargeting ${ }^{[10]}$. Different saliency models have been presented such as [11], where saliency means distinction from surroundings. As its higher dimensional counterpart, mesh saliency can be treated as region distinctiveness or importance in 3D. [2] develops a model of mesh saliency using center-surround filters with Gaussian-weighted curvatures. Their approach is explicitly based on the model of [11]. A similar saliency definition is presented in [12] based on local height variations and applied to surface feature line extraction. [3] proposes to use salient geometric features to do partial matching on triangular meshes. The salient features are extracted based on curvature and its variance. The curvature information is estimated from local quadric fitting, and no scale property of feature is considered here. To achieve the model's distinctiveness, [4] presents a method that not only considers the model alone, but also compares the model with other models in a database. This idea is unique but the availability of shape retrieval database suppresses its wider usage. A labeling and training based based feature prediction method is proposed in [5]. It is more related to the human perception but the

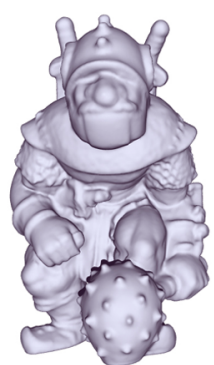

(a)

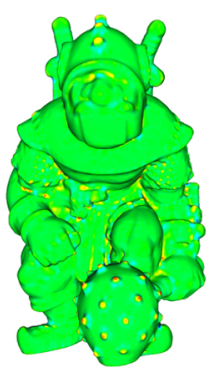

(b)

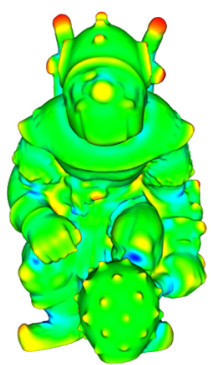

)

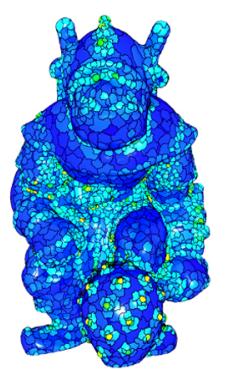

(c)

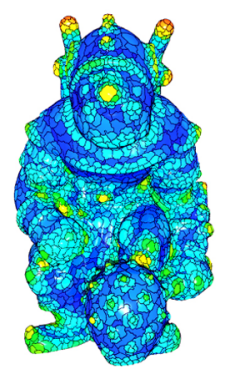

(c)

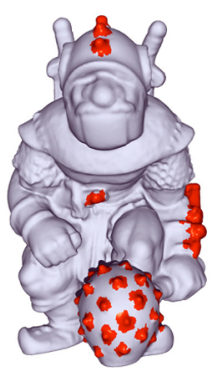

(d)

Fig.1. Multi-scale salient feature extraction. (a) Grog model. (b) Gaussian curvature on small and large scales. (c) Local surface descriptors on small and large scales. (d) Salient features extracted accordingly. 
scale at which the features are observed is not taken into account.

\subsection{Multi-Scale Feature Analysis}

Feature extraction has received a lot of attention in shape analysis direction due to the crucial information it contains. Moreover, feature has its scale specialty which corresponds to human perception and observation. The scale-space representations of $2 \mathrm{D}$ images $^{[13]}$ are defined as convolutions with Gaussian kernels of varying width $t$. This multi-scale image analysis tool has been extensively studied in computer vision community. Among these researches, [14] provides a method of automatic scale selection for reasonable feature analysis. The local appropriate scale is at which some normalized derivative operator assumes a local maximum. In computer graphics field, traditional multi-resolution geometry processing algorithms are based on hierarchical surface representations like progressive meshes $^{[15]}$ or subdivision surfaces ${ }^{[16]}$. [17] extracts a collection of piecewise linear curves describing the salient features of surfaces based on progressive mesh representations. [18] and [19] propose to use moment analysis of local surface patch neighborhood to do multi-scale shape editing. The process is based on a multi-scale feature guided surface decomposition and user interaction. [20] involves shape-space theory into multi-scale feature extraction on point-sampled surfaces. [21] uses multi-scale integral invariants and persistent feature in scale-space representations to robustly find feature point correspondences for global registration. Inspired by multi-scale analysis from computer vision community, [22] presents a geometric scalespace of 3D surface shapes using Ricci flow. The feature strength is measured by the magnitude of the Laplacian of the discrete Gaussian curvature. [23] defines heat diffusion process on a shape and the multi-scale property is reflected from the temporal domain of the heat kernel.

\section{Multi-Scale Salient Feature Extraction}

In this section, we present our multi-scale salient feature extraction algorithm in detail. For the geometric meaning of salient feature, we adapt to use the definition in [3], where salient feature is defined as compound high-level feature of non-trivial local shapes. Compared with the features represented per mesh vertex (cf. [2]), it conveys much more shape information of the local geometry. In their definition, the criterion of the salient local shape is related to its saliency and interestingness which is determined by curvature and its variance. However, to improve the robustness, the curvature information they used is an average from local fitting using different neighborhood sizes and no scale specialty is taken into account. In our paper, we propose to extract the salient geometric feature based on a robust multi-scale curvature estimation strategy. In this way, we can further judge the feature property whether it belongs to the surface detail or it represents surface more globally.

\subsection{Multi-Scale Curvature Estimation}

Instead of computing curvature based on local quadric fitting, we use multi-scale curvature estimation in [8]. The principal curvatures and the principal frame are estimated by principal component analysis (PCA) of local neighborhoods defined via spherical kernels centered on the given surface. As shown in Fig.2, suppose $p$ is a point on surface $S$. The intersection of the kernel ball $B_{r}$ or its boundary sphere $S_{r}$ with the interior (i.e., a locally defined side) of $S$ leads to the ball neighborhood $N_{b}^{r}$ and the sphere neighborhood $N_{s}^{r}$. The neighborhood radius $r$ can be naturally treated as the scale of interest. Here we only depict the sphere neighborhood case for more clear illustration purpose.

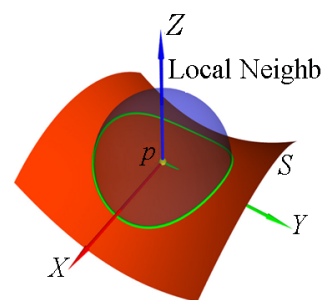

(a)

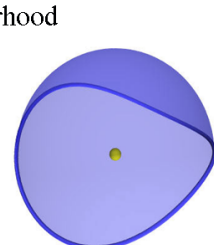

(b)

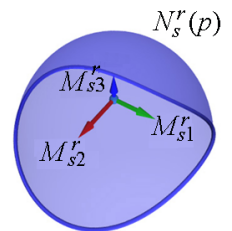

(c)
Fig.2. (a) Surface point and its spherical kernel. (b) Intersection part (sphere neighborhood here) which we perform PCA on. (c) Result of PCA: three moment vectors with different principal components.

We use PCA of the ball neighborhood for multi-scale principal curvature estimation all through this paper. For the implementation detail and complete theoretic analysis, we refer the readers to [9].

Fig. 3 shows the maximal principal curvature of the Asian Dragon model estimated on two different scales. Note that the scale features are more apparently recognized on the small scale compared with the dragon's back.

\subsection{Local Surface Descriptor Generation}

Based on the multi-scale curvature information which has been successfully estimated, a sparse set of local surface descriptors (LSD) will be built across the mesh surface afterwards. Each LSD is a surface point $p$ and its associated quadric patch that approximates the surface in a local neighborhood of $p^{[3]}$. This kind of 


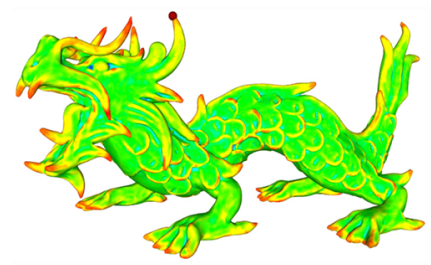

(a)

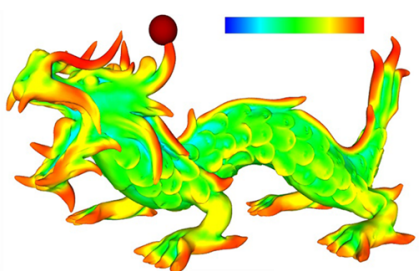

(b)
Fig.3. Multi-scale maximal principal curvatures on the Asian Dragon model, with two different kernels centered at one of its horns. The red color depicts the highest curvature value, blue color is for the lowest value. (a) Small scale. (b) Large scale.

LSD has many advantages: adaptive to the geometry of the shape, independent of the underlying triangulation, heavily reducing the complexity of the original triangle mesh representation, and ease of clustering non-trivial salient features.

In [3], the LSD of a surface point $p$ is built based on the geometric error between the local surface patch and the fitting quadric. However, in our method, the curvatures are estimated using PCA of local neighborhoods. In this case, the small shape variance can be neutralized on a large scale (see Fig.3), which means the geometry itself cannot reflect the change of the scale. So instead of using vertex coordinates, we build the LSD based on the curvature information, which is correlated with the scale of interest.

We also use the region-growing technique to iteratively generate the LSDs. First, we sort all the mesh vertices according to their curvature function value $\operatorname{Curv}(p)$ in descending order. The curvature function can be chosen depending on the model's property. Commonly we choose the absolute Gaussian curvature, and for CAD (computer aided design) models, the maximal absolute principal curvature will be used (see [3]). Then we build the LSDs one by one from the sorted list. For a vertex $p$ in the list which has not been in any LSD, we extract its associated quadratic patch in a way different from local fitting.

As discussed in Subsection 3.1, based on PCA of local neighborhood of a surface point $p$, we get three eigenvectors which form its local principal frame on scale $r$ besides principal curvatures $\kappa_{1}$ and $\kappa_{2}$. Then we form the paraboloid $P: z=\frac{1}{2}\left(\kappa_{1} x^{2}+\kappa_{2} y^{2}\right)$ in principal frame as the second order approximation of the surface at $p$ on the given scale. To generate the LSD from $p$, we greedily involve its neighbor vertices and integrate the error of Gaussian curvature over the local area until the prescribed threshold is reached. Suppose $q$ is one of its neighbors, we can get the local coordinates of $q$ by projecting it into $p$ 's principal frame. Then we only use the local $x, y$ coordinates $q_{x}$ and $q_{y}$ to compute the Gaussian curvature $\widehat{K}_{G}^{q}$ of the local osculating paraboloid as in (1).

$$
\widehat{K}_{G}^{q}=\frac{\kappa_{1} \kappa_{2}}{\left[1+\left(\kappa_{1} q_{x}\right)^{2}+\left(\kappa_{2} q_{y}\right)^{2}\right]^{2}}
$$

The error of the Gaussian curvature can then be estimated as the difference between $\widehat{K}_{G}^{q}$ and $K_{G}^{q}=\kappa_{1}^{q} \cdot \kappa_{2}^{q}$, where $\kappa_{1}^{q}$ and $\kappa_{2}^{q}$ are the principal curvatures of $q$ estimated in Subsection 3.1. Note that in this way, we do not involve local $z$ coordinate, and this can eliminate the error of LSD caused by the local shape variance when the scale becomes large. A $2 \mathrm{D}$ counterpart illustration is shown in Fig.4.

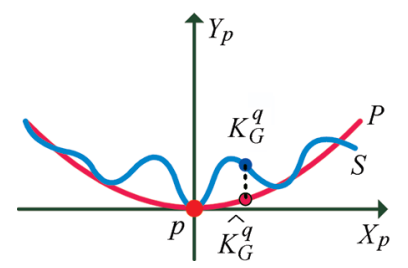

Fig.4. Estimating the curvature error of neighboring point $q$ from the osculating parabola of starting point $p$ in $2 \mathrm{D}$.

Note that when we accumulate the error of $q$, an area factor $\operatorname{Area}(q)$ is also involved. This is to compensate the error discrepancy if the same model has non-consistent tessellation. The area factor of a given vertex can be computed as $1 / 3$ of its one ring neighborhood.

In our implementation, for the model less than $100 \mathrm{~K}$ faces, we use 0.3 of its largest absolute Gaussian curvature times the average area per-vertex as the threshold. For large models, we increase the ratio to 1.0. This is due to the fact that the estimated curvature value will be depressed on a large scale. After a single LSD with starting point $p$ is extracted, we assign it with the largest curvature function value, i.e., $\operatorname{Curv}(p)$, as the representative curvature value.

Fig.5 shows the local surface descriptors of Gargoyle

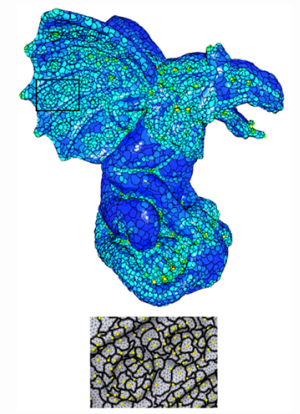

(a)

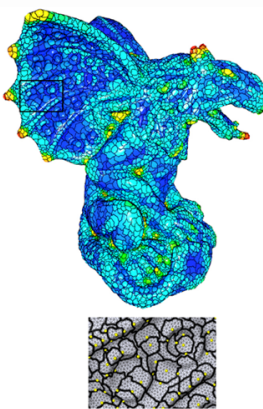

(b)
Fig.5. Local surface descriptors of Gargoyle model on two different scales. Red is high curvature function value and blue is low. Zoomed figures show the tiny structure and starting point of each LSD. (a) Small scale. (b) Large scale. 
model on two different scales. We can find the LSDs on small scale follow the surface detail (the ring-liked shape) better, while descriptors representing global curved shape are salient on large scale (see also zoom-in parts).

\subsection{Salient Feature Extraction}

The definition of saliency or salient feature is the foundation of distinctiveness analysis of $3 \mathrm{D}$ shapes. Due to its generality and our purpose of extracting multi-scale salient feature regions, we adapt to use the definition and measurement of salient feature in [3]. It defines salient feature as a cluster of LSDs that locally describes a non-trivial region of the surface.

For each LSD, we grow a cluster of descriptors by recursively adding its neighboring descriptors until the saliency grade of the clustered feature is maximized. This greedy process stops when the contribution of a candidate descriptor is insignificant. The saliency grade of a feature cluster is determined by the curvature function value of each LSD and its variance over the cluster. We refer readers to [3] for the details.

When the whole surface has been decomposed into feature clusters, the ones with high saliency grade will be extracted as salient geometric features. This can be done by a prescribed threshold of the saliency grade value or the percentage of salient features among all clusters. Since concave feature is usually generated by adjacent meaningful convex parts ${ }^{[24]}$, we suppress its saliency grade so that the inherent salient feature can be successfully extracted. The results of salient feature extraction of Grog model on two different scales can be found in Fig.1.

\section{Results and Discussion}

\subsection{Multi-Scale Feature Classification}

Feature classification is a high level content-based analysis of $3 \mathrm{D}$ shapes. The process is like building a

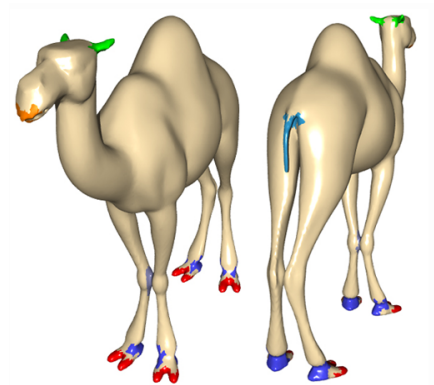

(a)

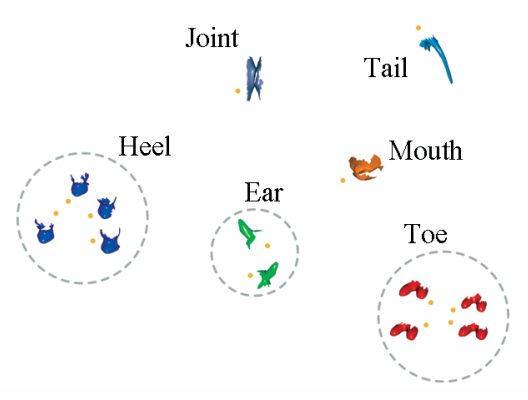

(b) group of equivalence classes on feature space. Features in the same class should have some specialized properties in common. This is very helpful for understanding the underlying geometry in a more global view. The classification results can be further applied to systematical feature editing and other applications ${ }^{[19,25]}$.

Our feature classification is based on multi-scale salient features extracted in Section 3. The goal is to classify salient features on different scales according to their global shapes, i.e., the salient features which have similar shape will be grouped into the same class. We believe this way of multi-scale salient feature extraction and classification will give us a comprehensive understanding of 3D models.

In our method, we use spin-image ${ }^{[26]}$ as the shape signature for each salient feature extracted from a 3D model. The resemblance between salient features is measured by their spin-images. A full distance matrix is generated afterwards. Then we extract a $2 \mathrm{D}$ embedding of the salient feature space using multidimensional scaling ${ }^{[27]}$. From the feature space, we obtain a meaningful classification of salient features.

Fig.6(a) shows the salient feature classification results of Camel model on small scale. We can see the meaningful body parts like ears, toes, heels, mouth, tail and joint of front legs are successfully classified as in Fig.6(b). The classification results on large scale of the same Camel model can be found in Fig.6(c). The salient features capture more global interesting shape of the surface. Toes and heels are merged to whole foot features.

Fig.7 shows the feature classification result of Grog model based on multi-scale salient feature extraction in Fig.1. We can find the sharp corners on the hammer are salient features only on small scale. And more global feature parts are extracted on large scale.

\subsection{Multi-Scale Viewpoint Selection}

Selecting a good viewpoint for 3D model has always

Fig.6. Feature classification of Camel model based on salient feature extraction on two different scales. (a) Classified salient features with different colors on small scale. (b) $2 \mathrm{D}$ projection of the salient feature space computed using classical multi-dimensional scaling. (c) Classified salient features on large scale. 


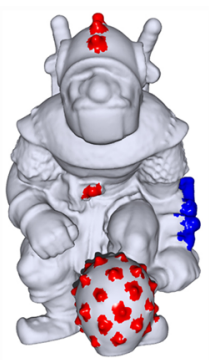

(a)

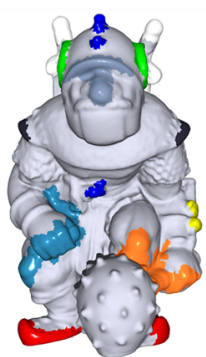

(b)
Fig.7. Feature classification of Grog model based on salient feature extraction on two different scales. (a) Classified salient features on small scale. (b) Result on large scale.

been an interesting and important problem. The information presented may differ considerably when a $3 \mathrm{D}$ model is viewed from different directions. Choosing a good viewpoint is also useful to applications such as generation of icons when browsing a large number of $3 \mathrm{D}$ models. In the process of selecting the optimal viewpoint, different properties of a 3D model can be taken into account. [2] develops a method for automatically selecting viewpoint so as to visualize the most salient object features. [28] introduces a method to choose good viewpoints by minimizing the symmetry observed from an object.

Based on our multi-scale salient feature extraction, we can do the viewpoint selection on multiple scales. In our approach, different viewpoints are determined by visible surface saliency on different scales. The visual effect is like observing an object from far to near. On large scale, features with some global shape information show up, while more details of an object are revealed on small scale. The intuition behind our approach is that people tend to notice global shape features of an object at first and then pay attention to more detailed ones. Thus, our approach helps to provide an informative illustration of a $3 \mathrm{D}$ object, with global and detailed features visible on different scales.

In our method, we define the saliency of a mesh vertex $v$ as $S(F) / \operatorname{Size}(F)$, where $F$ is the salient feature which contains vertex $v, S(F)$ is the saliency grade of $F$, Size $(F)$ is the number of vertices that belong to $F$. For vertex which does not belong to any salient feature, the saliency value is 0 . After that, we search for the viewpoint which maximizes the sum of saliency of all visible vertices. To avoid the sharp variance of saliency between neighboring viewpoints, here we set top $50 \%$ feature clusters as salient features.

Fig.8 shows the viewpoint-based saliency statistics result of Gargoyle model from uniform sampled directions. On large scale, the symmetry shape of the wireframe spherical shape indicates the global symmetry of the Gargoyle model. And for small scale, better

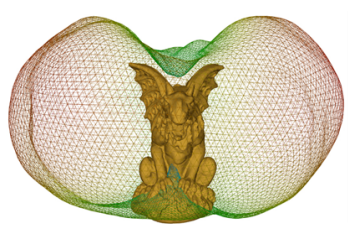

(a)

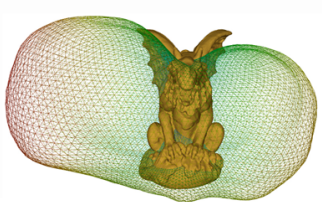

(b)
Fig.8. Multi-scale salient viewpoint selection for Gargoyle model. (a) Large scale. (b) Small scale.

viewpoint is located at the right side (corresponding to the model itself). This is because the tail of the Gargoyle which contains lots of surface details lies at right side.

The above viewpoint-based saliency computation is carried out on multiple scales so that different viewpoints can be selected. Fig.9 shows two optimal salient viewpoints of Gargoyle model, which are selected on large and small scale respectively. The upright orientation is manually selected here while automatic approach ${ }^{[29]}$ could also be involved. Note that two wings of Gargoyle model are more visible on large scale, while more attention is paid to detailed features such as rings and tail on small scale.

We also perform our multi-scale viewpoint selection algorithm on a CAD model as shown in Fig.10. It is easy to see that the viewpoints selected on two scales

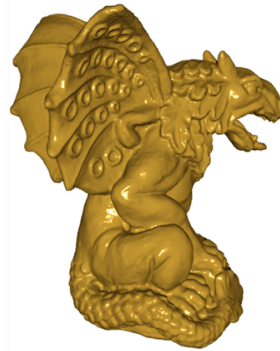

(a)

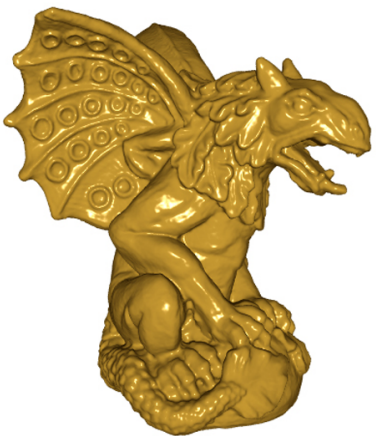

(b)
Fig.9. Multi-scale viewpoints selected on two different scales for Gargoyle model. (a) Large scale. (b) Small scale.

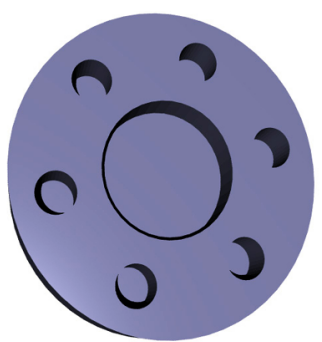

(a)

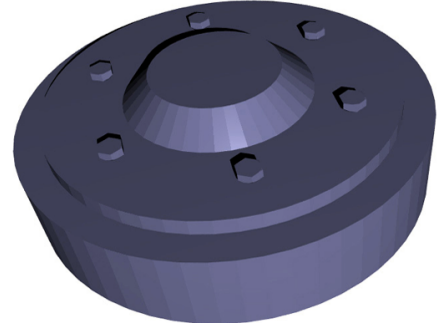

(b)
Fig.10. Multi-scale viewpoints selected on two different scales for a CAD model. (a) Large scale. (b) Small scale. 
are totally different. This is because features with different levels of details locate on different sides of the model. For small scale, the bolts are more salient on one side. On the opposite side, cylindrical hole features are preferred to be viewed on large scale. This viewpoint differences accord with human perception of recognizing the scale-dependent properties of a 3D model.

\subsection{Implementation Details}

Our multi-scale salient feature extraction algorithm is implemented in $\mathrm{C}++$ on the Windows platform. In all our experiments, we scale the models to fit into a bounding box of corner points $(-1,-1,-1)$ and $(1,1,1)$. On small scale, the radii of the ball neighborhood are set to 0.03 and on large scale it is $3 \sim 4$ times larger. For feature classification, we use the cmdscale function in Matlab to solve the multi-dimensional scaling and the kmeans function to perform a simple $K$ Means clustering. For viewpoint selection, we use the method in [30] to uniformly sample viewpoints on a sphere which encloses the bounding box of the model. Given a single viewpoint, the visible part of the model is extracted based on OpenGL depth buffer.

We test our multi-scale salient feature extraction algorithm on an Intel Core2 Duo $2.66 \mathrm{GHz}$ computer with 2GB RAM. For Camel model with $70 \mathrm{~K}$ triangles, the average cost of salient feature extraction on a single scale is 38.9 seconds. For Grog model with $200 \mathrm{~K}$ triangles, the cost is 40.5 seconds. The curvature estimation step takes most of the time. But when the model size grows, the cost is not increasing accordingly. This is because the curvature is computed by PCA of local ball neighborhood, and the computational complexity is determined by the voxelized grid which contains the model. This is very suitable for analysis of large models. For the details, we refer readers to [9]. On the other hand, the extraction of local surface descriptors and salient features are more efficient due to the greedy approach, and these two processes can be done within 5 seconds for all test models.

\subsection{Limitations and Discussion}

As shown in the previous experiments, our method can be used to extract salient features on different scales and analyse the shape at different levels of details. Compared with previous work ${ }^{[3]}$, instead of using moderate curvature information from averaging local fitting result with different neighborhood sizes, we perform integration-based robust curvature estimation on different scales. The extracted salient features coincide with the scale of interest and represent different characteristics of the shape. However, for a model with fruitful features, it will be very useful if we can identify the scale of the feature (i.e., what is the best dimension to measure the distinctiveness of the feature) and apply different scales to study different feature parts of the shape. Recent development on curvature estimation via adaptive integral invariants as in [31] may be used here.

Also, due to the simple region growing approach to extract salient feature parts, the boundary of the feature region is often jagged. A feature sensitive boundary smoothing ${ }^{[30]}$ or a graph-cut based algorithm ${ }^{[32]}$ can be used here as a post-processing step to improve the smoothness of the boundary.

\section{Conclusions and Future Work}

In this paper, we presented a new method of multiscale salient feature extraction. The salient features extracted on small scale represent the surface detail while more globally interesting salient regions can be extracted on large scale. This kind of multi-scale description of features accords with human perception from different scales of interest. We also applied the multi-scale salient feature extraction to feature classification and viewpoint selection, and both applications show that our method as a multi-scale analysis tool is very helpful for studying $3 \mathrm{D}$ shapes.

We want to apply the multi-scale salient feature extraction to a wider usage like shape matching, where different models can be compared on different scales. Models with details in common have more similarity on small scale while models with similar global shape regions are expected to be matched on large scale. We believe this kind of multi-scale feature based shape matching is favorable of further applications like modeling by example and shape retrieval.

\section{References}

[1] Tangelder J W, Veltkamp R C. A survey of content based 3D shape retrieval methods. In Proc. Shape Modeling and Applications 2004, June 2004, pp.145-156.

[2] Lee C H, Varshney A, Jacobs D W. Mesh saliency. ACM Trans. Graphics, 2005, 24(3): 659-666.

[3] Gal R, Cohen-Or D. Salient geometric features for partial shape matching and similarity. ACM Trans. Graphics, 2006, 25(1): 130-150.

[4] Shilane P, Funkhouser T. Distinctive regions of 3D surfaces. ACM Trans. Graphics, 2007, 26(2): Article No. 7.

[5] Chen X, Saparov A, Pang B et al. Schelling points on 3D surface meshes. Trans. Graphics, 2012, 31(4), Article No. 29.

[6] Yang Y L, Shen C H. Multi-scale salient feature extraction on mesh models. In Proc. Computational Visual Media Conference, Nov. 2012, pp.122-129.

[7] Gatzke T, Grimm C. Estimating curvature on triangular meshes. Int. J. Shape Modeling, 2006, 12(1): 1-28.

[8] Yang Y L, Lai Y K, Hu S M et al. Robust principal curvatures on multiple scales. In Proc. the 4th SGP, 2006, pp.223-226.

[9] Pottmann H, Wallner J, Yang Y L, Lai Y K, Hu S M. Principal curvatures from the integral invariant viewpoint. Computer 
Aided Geometric Design, 2007, 24(8-9): 428-442.

[10] Wang D, Li G, Jia W, Luo X. Saliency-driven scaling optimization for image retargeting. Visual Computer, 2011, 27(9): 853-860

[11] Itti L, Koch C, Niebur E. A model of saliency-based visual attention for rapid scene analysis. IEEE Trans. Pattern Analysis and Machine Intelligence, 1998, 20(11): 1254-1259.

[12] Miao Y, Feng J. Perceptual-saliency extremum lines for 3Dd shape illustration. Visual Computer, 2010, 26(6-8): 433-443.

[13] Witkin A P. Scale-space filtering. In Proc. the 8th Int. Joint Conf. Artifical Intelligence, Aug. 1983, Vol.2, pp.1019-1022.

[14] Lindeberg T. Feature detection with automatic scale selection. Int. J. Computer Vision, 1998, 30(2): 79-116.

[15] Hoppe H. Progressive meshes. In Proc. the 23rd SIGGRAPH, Aug. 1996, pp.99-108.

[16] Zorin D, Schröder P, Sweldens W. Interactive multiresolution mesh editing. In Proc. the 24th SIGGRAPH, Aug. 1997, pp.259-268.

[17] Hubeli A, Gross M. Multiresolution feature extraction for unstructured meshes. In Proc. Visualization 2001, Oct. 2001, pp.287-294

[18] Clarenz U, Rumpf M, Telea A. Robust feature detection and local classification for surfaces based on moment analysis. IEEE Transactions on Visualization and Computer Graphics, 2004, 10(5): 516-524.

[19] Clarenz U, Griebel M, Rumpf M, Schweitzer M A, Telea A. Feature sensitive multiscale editing on surfaces. Visual Computer, 2004, 20(5): 329-343.

[20] Pauly M, Keiser R, Gross M. Multi-scale feature extraction on point-sampled surfaces. Computer Graphics Forum, 2003, 22(3): 281-289.

[21] Gelfand N, Mitra N J, Guibas L J, Pottmann H. Robust global registration. In Proc. the 3rd SGP, July 2005, pp.197-206.

[22] Zou G, Hua J, Lai Z, Gu X, Dong M. Intrinsic geometric scale space by shape diffusion. IEEE Transactions on Visualization and Computer Graphics, 2009, 15(6): 1193-1200.

[23] Sun J, Ovsjanikov M, Guibas L. A concise and provably informative multi-scale signature based on heat diffusion. Computer Graphics Forum, 2009, 28(5): 1383-1392.

[24] Katz S, Tal A. Hierarchical mesh decomposition using fuzzy clustering and cuts. Trans. Graphics, 2003, 22(3): 954-961.

[25] Lai Y K, Zhou Q Y, Hu S M, Wallner J, Pottmann H. Robust feature classification and editing. IEEE Transactions on Visualization and Computer Graphics, 2007, 13(1): 34-45.
[26] Johnson A. Spin-Images: A representation for 3-D surface matching [Ph.D. Thesis]. Robotics Institute, Carnegie Mellon University, Pittsburgh, USA, August 1997.

[27] Cox T, Cox M. Multidimensional Scaling (2nd edition). London, UK: Chapman \& Hall, 2001.

[28] Podolak J, Shilane P, Golovinskiy A, Rusinkiewicz S, Funkhouser T. A planar-reflective symmetry transform for 3D shapes. ACM Trans. Graphics, 2006, 25(3): 549-559.

[29] Fu H, Cohen-Or D, Dror G et al. Upright orientation of manmade objects. Trans. Graphics, 2008, 27(3), Article No. 42.

[30] Lai Y K, Zhou Q Y, Hu S M, Wallner J, Pottmann H. Robust feature classification and editing. IEEE Transactions on Visualization and Computer Graphics, 2007, 13(1): 34-45.

[31] Lai Y K, Hu S M, Fang T. Robust principal curvatures using feature adapted integral invariants. In Proc. SPM 2009, Oct. 2009, pp.325-330.

[32] Yan D M, Wang W P, Liu Y, Yang Z. Variational mesh segmentation via quadric surface fitting. Computer-Aided Design, 2012, 44(11): 1072-1082.

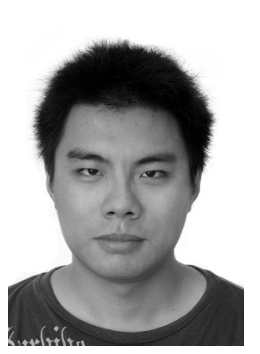

Yong-Liang Yang received his Bachelor's and Ph.D. degrees in computer science from Tsinghua University, Beijing, in 2004 and 2009, respectively. He is currently a research scientist in Geometric Modeling and Scientific Visualization Center, King Abdullah University of Science and Technology, Saudi Arabia. His research interests include computer graphics, geometric modeling and geometry processing.

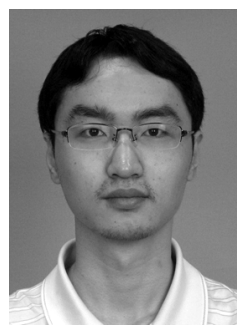

Chao-Hui Shen received the Bachelor's degree in computer science from Tsinghua University, Beijing, in 2008. He is currently a Ph.D. candidate in the Department of Computer Science and Technology, Tsinghua University. His research interests include computer graphics, geometric modeling and processing. 